\title{
A strategy for using cooperating missiles for missile defence
}

\author{
M. Kapsis $^{a}$ (D), P. Pudney ${ }^{\text {a }}$ (D), J. Aarao ${ }^{a}$ (D), W. Miller ${ }^{b}$ (iD) and G. Freebairn ${ }^{b}$ \\ ${ }^{a}$ University of South Australia, GPO BOX 2471, Adelaide, South Australia, 5001 \\ ${ }^{\mathrm{b}}$ DST Group, PO BOX 1500, Edinburgh, South Australia, 5111 \\ Email: maria.kapsis@mymail.unisa.edu.au
}

\begin{abstract}
We are interested in exploring the concept of using a team of low-cost defending missiles instead of a single high-cost defending missile to intercept a high-performance incoming missile. The low-cost defending missiles will have reduced seeker performance or reduced range or manoeuvrability compared to a high-cost defending missile, but will cooperate with each other to seek and intercept the incoming missile.

This paper presents a cooperative control strategy for a team of cooperating defending missiles. The objective is to minimise the probability of missing the incoming missile.

We formulate a method to predict possible paths of the incoming missile from a known initial state to a known target, and introduce the concepts of a seek region and intercept region that can be used to determine points for the defending missiles to aim towards before operating their on-board seekers. These aim points are placed such that the defenders are able to maximise their coverage of the seek and intercept regions within the incoming missile's manoeuvrability range. We demonstrate a simple method for calculating aim points with a couple of examples.
\end{abstract}

Keywords: Cooperative control, team defence, air missile defence systems 


\section{INTRODUCTION}

The motivation for our work is to determine whether replacing a single high-cost defence missile with a team of cooperating low-cost defence missiles can improve performance and flexibility of missile defence systems and be more cost effective. Low-cost defence missiles are likely to have reduced seeker performance or reduced range or manoeuvrability compared to a high-cost defence missile, but can work as a team to seek and intercept an incoming missile.

In this paper we develop a strategy for coordinating a team of defending missiles. In particular, we develop a method for predicting the possible paths of an incoming missile and then determining where the defending missiles should fly towards so that they can effectively detect and intercept the incoming missile.

\subsection{The Missile Defence System}

Figure 1 depicts the components that make up a missile defence system. A typical mission timeline is:

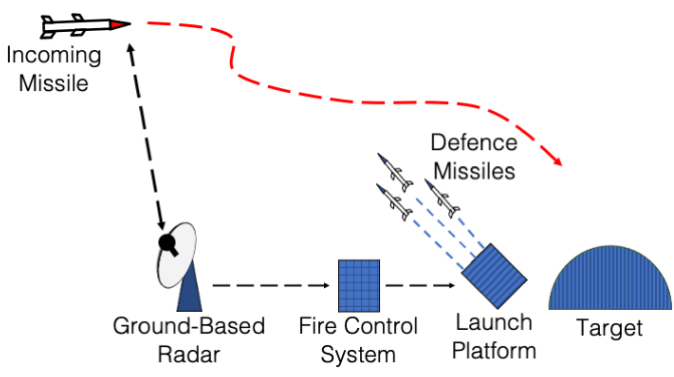

Figure 1. Components of the missile defence system

1. The incoming missile is detected by the ground-based radar.

2. The ground-based radar will track the incoming missile and provide position, speed and heading measurements to the fire control system. We assume that the ground-based radar will be able to send data to the defending missiles before and after launch.

3. The fire control system will determine how many defending missiles to launch, when to launch them, and where each defending missile should fly to before it turns on its seeker.

4. As the defending missiles fly out towards the incoming missile, information from the ground-based radar will be used to refine the possible paths of the incoming missile, which in turn will be used to refine the points that the defending missiles are flying towards.

5. Each defending missile will be equipped with a seeker that can detect the incoming missile, but the seeking angle and seeking range may be significantly reduced in comparison to a high-performance defending missile. Once the defending missiles are within seeking range of the incoming missile, they will turn on their on-board seekers. At this stage, the defending missile positions and directions should be organised so that their seekers cover all possible paths of the incoming missile.

6. Low-cost defending missiles may have limited abilities to pursue an incoming missile, but they will have the ability to cooperate with each other. Once the incoming missile is detected by any defending missile, the team of defending missiles will coordinate to track and intercept the incoming missile with as many defending missiles as possible.

\subsection{Cooperative control strategies}

Su et al. $|2018|$ describe a cooperative control strategy to guide multiple inferior missiles to cooperatively cover the incoming missile maneuverability range. They assume acceleration limits and divide the incoming missile's acceleration commands into sections and assign defending missiles to cover each section. Their guidance goal is to cover as much of the possible maneuverability range as possible. Defending missiles communicate during flight and adapt their covering strategy against evasive strategies such as constant manoeuvring, 
random step manoeuvring and bang-bang manoeuvring. Su et al. [2018] claim that their adaptive covering strategy gives smaller miss distance - the minimum distance between the defending missile and the incoming missile - and higher successful interception probability - coverage of the predicted intercept region-than that of augmented proportional navigation guidance [Garber, 1968] and differential game guidance [Gutman and Leitmann, 1976; Shinar and Gutman, 1980| when used by a team of three defending missiles. Su et al. |2018| consider defending missiles with inferior maneuverability, but do not consider limitations of information from a ground-based radar or inferior performance of on-board seekers. Additionally Rajagopalan et al. |2019| apply the Su et al. [2018] formulation and find that the cooperative control strategy is not effective against an incoming missile capable of high-acceleration terminal maneuvering.

Rajagopalan et al. [2019] use a predictive regret-matching algorithm to guide a team of defending missiles to totally cover the incoming missile's reachability. Rather than dividing the acceleration limits of the incoming missiles into a number of regions for the defenders to cover, they first project future positions of the incoming missile based on its speed and acceleration limits, then coordinate the defenders to cover off the incoming missile's reachability. Once the incoming missile's reachability is totally covered by the team of defenders then the proportional navigation guidance law is used to steer the defending missiles to intercept the incoming missile. A clear difference between this approach and that of Su et al. |2018| is that Su et al. [2018| does not transition the defending missiles to a different guidance law to intercept the incoming missile. Rajagopalan et al. [2019] assume that the defending missiles can see the incoming missile for the entire duration of their flight - they do not model a separate seeking phase where the defenders rely on a ground-based radar to provide updates of the incoming missile's position and velocity.

\subsection{Overview of the paper}

In this paper we formulate a method to predict possible paths of the incoming missile from a known initial state to a known target (Section 2.1), and introduce the concepts of a seek region (Section 2.2) and intercept region (Section 2.3) that can be used to determine points for the defending missiles to aim towards before operating their on-board seekers (Section 2.4). These aim points are placed such that the defenders are able to maximise their coverage of the seek and intercept regions within the incoming missile's manoeuvrability range. We demonstrate a simple method for calculating aim points with a couple of examples (Section 3 ).

\section{Problem Formulation}

We consider a simplified 2D problem where missile paths are constrained to a vertical plane and the curvature of the Earth is ignored. Our methodology could be extended to a 3D problem as the incoming missile paths, defender paths, the seek region, the intercept region and the aim points could all be formulated in 3D.

We want to defend against a single incoming missile that is assumed to fly towards a target at position $(0,0)$. Suppose the incoming missile is detected at time $t_{0}$ by a ground-based radar to be at position $P_{0}$ with velocity vector $\boldsymbol{v}_{A}$. The incoming missile is able to perform aerodynamic manoeuvres, thus its future path towards the target is uncertain. We have up to $N$ defence missiles that can cooperate together to seek and intercept the incoming missile. Our method for determining how to defend against the incoming missile has four stages:

1. determine the possible paths of the incoming missile towards the target

2. define a seek region comprising points in time and space where the incoming missile could be when it is first detected by defending missile seekers

3. define an intercept region comprising points in time and space where the incoming missile could be when it is intercepted

4. define aim points that the defending missiles will fly towards so that they can effectively seek and intercept the incoming missile.

Prior to launching the defending missiles, and during their fly-out phase, the ground-based radar will provide periodic updates on the incoming missile's position and velocity vector, and so the incoming missile's paths, the seek region, the intercept region and the aim points will change. The challenge is to find aim points that will maximise the probability of intercepting the incoming missile and ensure the defending paths remain feasible. 


\subsection{Predicting Incoming Missile Paths}

An incoming missile path will be a smooth curve that terminates at the target location. Missiles are limited in their ability to manoeuvre by aerodynamic and structural constraints |Raymer, 1992|, which define the maximum curvature of the path. We use cubic Bezier curves [Hazewinkel, 1997| to represent incoming missile paths as they are smooth and easy to use. These parametric curves have the form

$$
B(t)=\sum_{k=1}^{3}\left(\begin{array}{l}
3 \\
k
\end{array}\right) t^{k}(1-t)^{3-k} P_{k}, \quad t \in[0,1]
$$

where the control points are:

$P_{0}$ : the initial position of the incoming missile, detected by the radar

$P_{1}$ : a point ahead of the missile that is on the line through the current missile position in the current missile direction

$P_{2}$ : a point that defines the direction from which the missile will approach the target and the approach path

$P_{3}$ : the position of the target.

We generate different paths by modifying $P_{1}$ and $P_{2}$. We assume that the incoming missile will travel at a constant speed throughout its flight. Figure 2 shows a set of 500 random missile paths (orange lines) for a missile travelling at $\boldsymbol{v}_{A}=\left(-600 \mathrm{~ms}^{-1}, 0 \mathrm{~ms}^{-1}\right)$ detected at $P_{0}=(30000 \mathrm{~m}, 6000 \mathrm{~m})$ (red dot). Paths with curve radius less than $3670 \mathrm{~m}$ have been discarded corresponding to a maximum acceleration limit of $10 \mathrm{~g}$. The actual path of the incoming missile (red line) will be unknown to the defending missile team. The black dots indicate the incoming missile position at 3 -second intervals along each path.

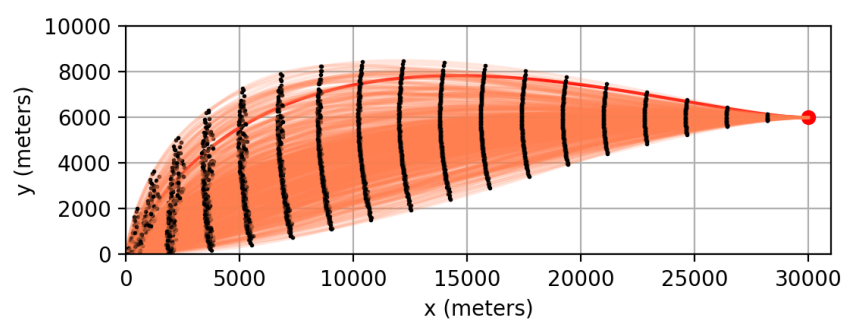

Figure 2. 500 possible incoming missile paths

\subsection{Seek Region}

We start by choosing a seek region. This is the region where the incoming missile could be when it is first detected by the defending missile seekers. The location of the seek region should be:

- within the detection range of the ground-based radar

- far enough from the target so that the defending missiles have enough time to fly to within seeking range of the seek region before the incoming missile reaches the seek region, and

- close enough to the last known position of the incoming missile such that the extent of the seek region is not too large.

The blue dots in Figure 3a show a seek region at $x=20000 \mathrm{~m}$. The incoming missile is at $P_{0}=$ $(30000 \mathrm{~m}, 6000 \mathrm{~m})$, and will take between 15 and 18 seconds to reach the seek region. Defending missiles travelling at $900 \mathrm{~ms}^{-1}$ and with a seek range of $10000 \mathrm{~m}$ will require about 11 seconds to fly to around $x=10000 \mathrm{~m}$, from where they can start seeking. Figure $3 \mathrm{~b}$ shows the portion of the seek region that is covered by a defender at $x=11000 \mathrm{~m}$ with a seeker view angle of $\pm 5^{\circ}$ and a detection range of $10000 \mathrm{~m}$. In this example, we will need at least two defending missiles to cover the entire seek region shown in Figure $3 \mathrm{a}$ We need to choose where each defending missile will be positioned and its direction of travel when it turns on its seeker. Furthermore, these aim points (which incorporate time, location, and direction) must be achievable from the defender launch position. The desired arrival time of each defender at its aim point is determined by the time it will take the incoming missile to reach the seek region. 
M. Kapsis et al., A strategy for using cooperating missiles for missile defence

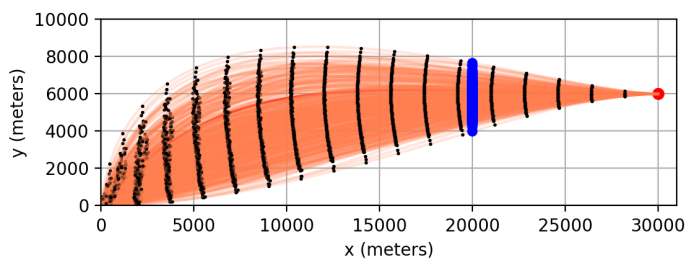

(a) Seek region at $x=20000 \mathrm{~m}$

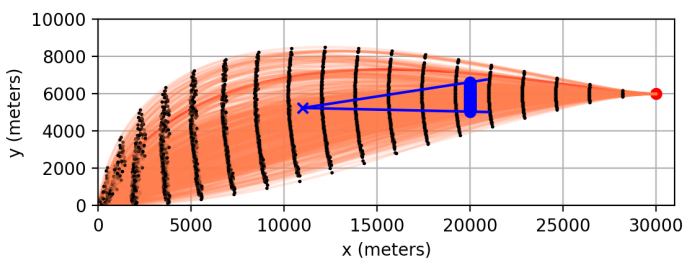

(b) Region seen by a defending missile at $x=11000 \mathrm{~m}$

Figure 3: Seek region

\subsection{Intercept Region}

When choosing the aim points for the defending missiles, we want to choose aim points that cover the seek region but also points from which it is possible to intercept the incoming missile with as many defending missiles as possible. The intercept region will lie between the aim points and the seek region. For each possible incoming missile path and each aim point, we can calculate whether or not the defending missile can intercept the incoming missile and, if it can, where and when the intercept will occur. For our example problem, if the defending missiles are at $x=11000 \mathrm{~m}$ and see the incoming missiles at $x=20000 \mathrm{~m}$ then, based on the relative speeds of the incoming missile and the defending missiles, the intercepts will be at approximately $x=16400 \mathrm{~m}$. This region is shown in Figure $4 \mathrm{a}$ by the red crosses. A defending missile at an aim point may not be able to cover the entire intercept region. We know the time, position and direction of each aim point, as well as the speed and maximum angular velocity of the defending missiles, so we can calculate which portion of the intercept region is covered by each aim point. Figure $4 \mathrm{~b}$ shows the portions of the seek region and the intercept region that will be covered by a particular aim point.

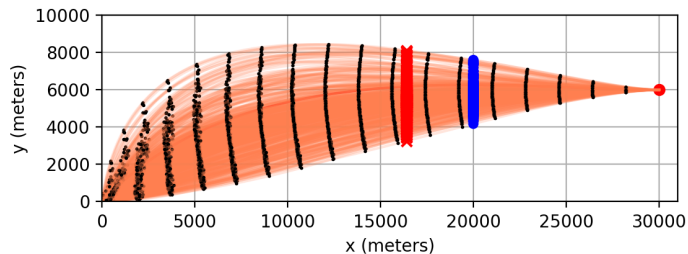

(a) Intercept region at $x=16400 \mathrm{~m}$

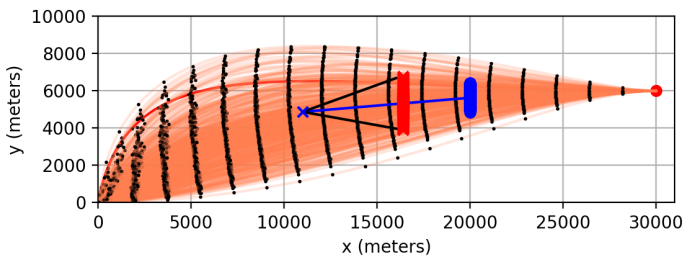

(b) Region covered by a defending missile at $x=11000 \mathrm{~m}$

Figure 4. Intercept region

\subsection{Aim points}

Our objective is to find aim points for multiple defending missiles that cover as much of the seek and intercept regions as possible. The aim point for each defending missile specifies the time, location and direction of the defending missile when it turns on its seeker. We can generate effective aim points using the following method:

1. choose a seek region, construct the intercept region and determine the number of defending missiles $n \leq N$ that is required to cover the seek and intercept region,

2. partition the seek and intercept regions into $n$ equal sub-regions,

3. for each defending missile $i$, construct a direction vector that passes through the centers of intercept sub-region $i$ and seek sub-region $i$.

The method is illustrated in Figure 5, where the aim points (blue crosses) are placed such that the centers of the seek and intercept sub-regions are covered. This method is not necessarily optimal-we discuss optimisation in Section 4. 
M. Kapsis et al., A strategy for using cooperating missiles for missile defence

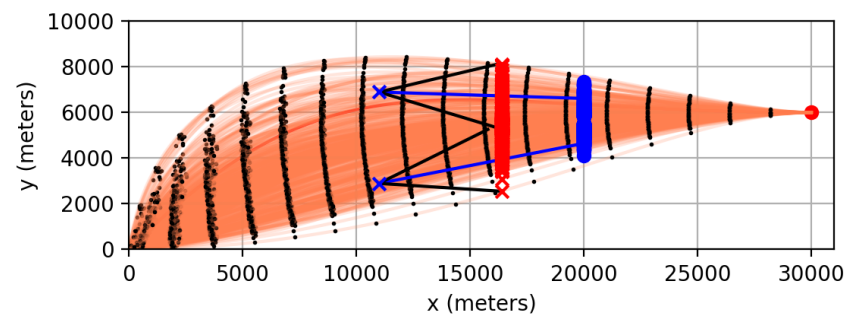

Figure 5. Example of two aim points (blue crosses) placed such that they cover the seek and intercept regions

\section{EXAMPLES}

We illustrate the feasibility of our approach with two examples, the first with one defending missile and the second with two defending missiles. These examples show how the seek region, intercept region and aim points evolve as information about the incoming missile is updated from the ground-based radar.

The first example (Figure 6) has a single defending missile. The defending missile was launched about 3 seconds before time $t_{1}$, and is at $(2500 \mathrm{~m}, 1500 \mathrm{~m})$ at time $t_{1}$. At time $t_{1}$ (Figure 6a) there is a radar update. The new position of the incoming missile is $P_{1}=(27000 \mathrm{~m}, 6000 \mathrm{~m})$, about 11 seconds before it reaches the seek region at $x=20000 \mathrm{~m}$. We recalculate the possible incoming paths, find the centres of the seek and intercept regions, and use these to calculate a new aim point at which we will turn on the defender's seeker. The new aim point is at $(11000 \mathrm{~m}, 5500 \mathrm{~m})$. From this aim point the defender can cover almost the entire seek region, and most of the intercept region. The defender path from the current location at (2500 m, $1500 \mathrm{~m})$ to the new aim point can be found using a Dubins path [Dubins, 1957], which is the shortest path that respects the minimum turn radius of the defending missile. Figure $6 \mathrm{~b}$ shows the situation when the radar updates again at time $t_{2}$, about 4 seconds later. The incoming missile is now about 6 seconds from the seek region. The seek region and intercept region are both smaller, because the incoming missile is closer and has less time to manoeuvre. We calculate a new aim point at $(11000 \mathrm{~m}, 5700 \mathrm{~m})$, and a new Dubins path from the current defender position at $(4500 \mathrm{~m}, 2750 \mathrm{~m})$ to the new aim point. The new aim point covers all of the seek region and all of the intercept region.

Once the incoming missile has been detected by one of the defending missile seekers, we assume the position of the incoming missile can be continuously shared amongst the defending missiles and each defending missile can use a standard intercept strategy, such as proportional navigation, to intercept the incoming missile. Figure 7 shows an example where two defending missiles were launched. In this case the two missiles are able to cover the entire seek region and the entire intercept region.

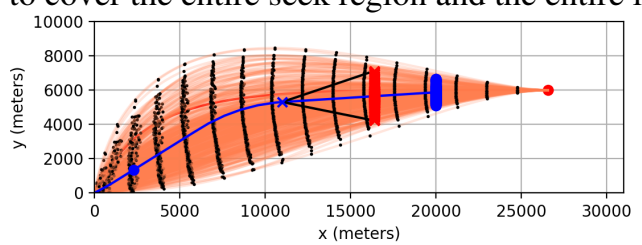

(a) Predicted paths at time $t_{1}$

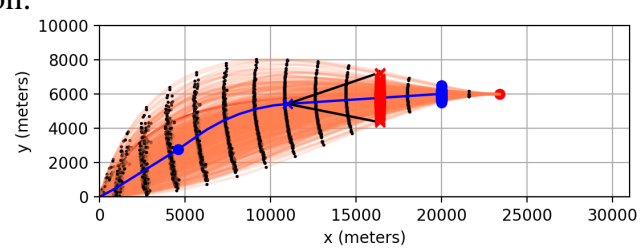

(b) Predicted paths at time $t_{2}$

Figure 6. Example with one defender

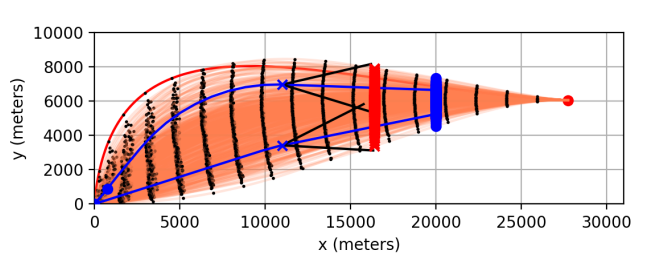

(a) Predicted paths at time $t_{1}$

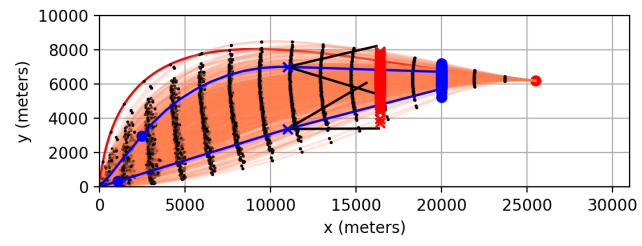

(b) Predicted paths at time $t_{2}$

Figure 7. Example with two defenders 


\section{CONCLUSions AND Future Work}

We have introduced concepts for constructing a cooperative control strategy for a team of defending missiles to cover a seek region and an intercept region that takes into account the maneuverability range of an incoming missile. We have used a simple method to calculate aim points that define the time, location and direction that each defending missile should fly to before turning on its on-board seeker. We have given two examples that show how our method would work in practice. However, there is scope for optimising the way that the aim points are calculated. Optimal aim points would:

- maximise the coverage of the seek region, so that the probability of detecting the incoming missile is maximised

- maximise the coverage of the intercept region, so that as many defending missiles as possible can intercept the incoming missile

- ensure that the sequence of aim points remains effective as they are updated from periodic radar measurements of the incoming missile.

Once we have demonstrated effective strategies for using cooperating teams of defending missiles, we can evaluate the scope for replacing high-cost and high-performance defence missiles with multiple cooperating low-cost and low-performance missiles to improve performance, flexibility and cost effectiveness of missile defence systems.

\section{ACKNOWLEDGMENTS}

This project is being conducted as part of the Department of Defence STEM Cadetship Program. We thank the team at DST Group for modelling advice.

\section{REFERENCES}

Dubins, L. E. [1957], 'On Curves of Minimal Length with a Constraint on Average Curvature, and with Prescribed Initial and Terminal Positions and Tangents', American journal of mathematics 79(3), 497-516. Publisher: Johns Hopkins Press.

Garber, V. [1968], 'Optimum intercept laws for accelerating targets.', AIAA Journal 6(11), 2196-2198. Publisher: American Institute of Aeronautics and Astronautics.

URL: https://doi.org/10.2514/3.4962

Gutman, S. and Leitmann, G. [1976], 'Optimal strategies in the neighborhood of a collision course', AIAA Journal 14(9), 1210-1212. Publisher: American Institute of Aeronautics and Astronautics.

URL: https://doi.org/10.2514/3.7213

Hazewinkel, M. [1997], Encyclopaedia of Mathematics: Supplement Volume I, Dordrecht: Springer Netherlands, Dordrecht.

Rajagopalan, A., Nguyen, D. D. and Kim, J. [2019], 'Predictive Regret-Matching for Cooperating Interceptors to Defeat an Advanced Threat', pp. 28-40. Place: Cham Publisher: Cham: Springer International Publishing.

Raymer, D. P. [1992], Aircraft Design: A Conceptual Approach, 2 edn, American Institue of Aeronautics and Astronautics, Washington, DC.

Shinar, J. and Gutman, S. [1980], 'Three-dimensional optimal pursuit and evasion with bounded controls', IEEE transactions on automatic control 25(3), 492-496. Publisher: IEEE.

Su, W., Shin, H.-S., Chen, L. and Tsourdos, A. [2018], 'Cooperative interception strategy for multiple inferior missiles against one highly maneuvering target', Aerospace science and technology 80, 91-100. Publisher: Elsevier Masson SAS. 